\title{
Agonist-induced formation of FGFR1 homodimers and signaling differ among members of the FGF family
}

\author{
Wilber Romero-Fernandez ${ }^{\mathrm{a}}$, Dasiel O. Borroto-Escuela ${ }^{\mathrm{a}}$, Alexander O. Tarakanov ${ }^{\mathrm{b}}$, Giuseppa Mudó ${ }^{\mathrm{c}}$, \\ Manuel Narvaez ${ }^{\mathrm{d}}$, Mileidys Pérez-Alea ${ }^{\mathrm{e}}$, Luigi F. Agnati ${ }^{\mathrm{f}}$, Francisco Ciruela ${ }^{\mathrm{g}}$, Natale Belluardo ${ }^{\mathrm{c}}$, Kjell Fuxe ${ }^{\mathrm{a}, *}$ \\ ${ }^{a}$ Department of Neuroscience, Karolinska Institutet, Stockholm, Sweden \\ ${ }^{\mathrm{b}}$ Russian Academy of Sciences, St. Petersburg Institute for Informatics and Automation, Saint Petersburg, Russia \\ ${ }^{\mathrm{c}}$ Department of Experimental Biomedicine and Clinical Neurosciences, Division of Human Physiology, Laboratory of Molecular Neurobiology, University of Palermo, \\ Corso Tukory 129, 90134 Palermo, Italy \\ ${ }^{\mathrm{d}}$ Department of Physiology, School of Medicine, University of Málaga, Spain \\ e School of Life and Health, Aston University, Birmingham, United Kingdom \\ ${ }^{\mathrm{f}}$ IRCCS, Lido Venice, Italy \\ ${ }^{\mathrm{g}}$ Unitat de Farmacologia, Departament Patologia i Terapèutica Experimental, Universitat de Barcelona, Spain
}

\section{A R T I C L E I N F O}

\section{Article history:}

Received 12 May 2011

Available online 20 May 2011

\section{Keywords:}

Fibroblast growth factors

FGFR1

Homodimerization

BRET

MAPK

\begin{abstract}
A B S T R A C T
Fibroblast growth factor receptor 1 (FGFR1) is known to be activated by homodimerization in the presence of both the FGF agonist ligand and heparan sulfate glycosaminoglycan. FGFR1 homodimers in turn trigger a variety of downstream signaling cascades via autophosphorylation of tyrosine residues in the cytoplasmic domain of FGFR1. By means of Bioluminescence Energy Resonance Transfer (BRET) as a sign of FGFR1 homodimerization, we evaluated in HEK293T cells the effects of all known FGF agonist ligands on homodimer formation. A significant correlation between BRET ${ }^{2}$ signaling and ERK1/2 phosphorylation was observed, leading to a further characterization of the binding and signaling properties of the FGF subfamilies. FGF agonist ligand-FGFR1 binding interactions appear as the main mechanism for the control of FGFR1 homodimerization and MAPK signaling which demonstrated a high correlation. The bioinformatic analysis demonstrates the interface of the two pro-triplets SSS (Ser-Ser-Ser) and YGS (Tyr-Gly-Ser) located in the extracellular and intracellular domain of the FGFR1. These pro-triplets are postulated participate in the FGFR1 homodimerization interface interaction. The findings also reveal that FGF agonist ligands within the same subfamily of the FGF gene family produced similar increases in FGFR1 homodimer formation and MAPK signaling. Thus, the evolutionary relationship within this gene family appears to have a distinct functional relevance.
\end{abstract}

(c) 2011 Elsevier Inc. All rights reserved.

\section{Introduction}

The FGFR1 plays important roles in several processes of the life cycle, including cell proliferation, differentiation, and apoptosis [1]. Fibroblast growth factors (FGFs) bind to the extracellular binding pocket of FGFR1 promoting receptor dimerization and autophosphorylation of tyrosine residues in the receptor cytoplasmic domain [2]. FGF signaling is strongly regulated at the level of the extracellular receptor-ligand complex assembly by spatial and temporal expression of ligands, heparan sulfate proteoglycans,

\footnotetext{
* Corresponding author. Address: Department of Neuroscience, Karolinska Institutet, Retzius vag 8, 17177 Stockholm, Sweden. Fax: +46 8315721.

E-mail addresses: Wilber.Romero-Fernandez@ki.se (W. Romero-Fernandez) Dasiel.Borroto-Escuela@ki.se (D.O. Borroto-Escuela), g.mudo@unipa.it (G. Mudó), mnarvaez@uma.es (M. Narvaez), m.perez@aston.ac.uk(M. Pérez-Alea), luigiagnati@ tin.it (L.F. Agnati), fciruela@ub.edu (F. Ciruela), n.belluardo@unipa.it (N. Belluardo), Kjell.Fuxe@ki.se (K. Fuxe).
}

receptors and also via intracellular modulation of downstream effectors [3]. However, FGF ligand-FGFR1 binding properties are the most critical mechanism for the control of FGFR1 signaling. FGFR1 binds members of the FGF family with different affinities and capabilities to produce activation, and alternative mRNA splicing leads to isoforms of FGFR1 having special ligand binding properties [4]. In addition, FGF ligand/FGF ligand and FGFR1/FGFR1 dimerization may expand the spectrum of interactions between FGFs and FGFR affecting their binding and signaling properties [5]. Anomalous FGF signaling is associated with several human diseases, for instance uncontrolled FGF signaling has been linked to the etiology of neurodegenerative disorders, such as major depressive disorder and Parkinson's disease [6].

In this paper we have studied the agonist-induced rise of FGFR homodimerization using BRET ${ }^{2}$ as a sign of FGFR1 binding. We have evaluated the effects of all known FGF ligands on FGFR1FGFR1 homodimer formation by means of BRET ${ }^{2}$ and significantly 
correlated this action to ERK1/2 phosphorylation leading to a further characterization of the binding and signaling properties of the FGF subfamilies.

\section{Materials and methods}

\subsection{Receptor constructs}

The FGFR1 tagged gene was amplified from the pcDNA3.1+ -FGFR1 vector (imaGenes GMBH, Berlin, Germany) using sense oligonucleotides primer Fw-BglII-FGFR1 (5'-GAAGATCTTCCAAAAT GTCGTAACAACTCCGCC- $3^{\prime}$ ) and antisense primer Rv-BamHI-5HT1A (5'-CGGGATCCCGCCATTGGCAAGCTGG - $3^{\prime}$ ) resulting in a cDNA fragment of $2199 \mathrm{bp}$, subcloned into the $\mathrm{pGFP}^{2}-\mathrm{N} 1$ (Perkin-Elmer, Spain) and humanized pRluc-N3 (Packard Bioscience, Spain). The reading frame and PCR integrity of all cloned constructs were confirmed by DNA sequencing.

\subsection{Cell culture and transfection}

HEK293T cells (American Type Culture Collection, USA) were grown in Dulbecco's modified Eagle's medium supplemented with $2 \mathrm{mM}$ L-glutamine, 100 units/ml penicillin/streptomycin, and $10 \%$ $(\mathrm{v} / \mathrm{v})$ fetal bovine serum at $37^{\circ} \mathrm{C}$ and in an atmosphere of $5 \% \mathrm{CO}_{2}$. For transfection, the cells were plated in six-well dishes at a concentration of $1 \times 10^{6}$ cells/well or in $75 \mathrm{~cm}^{2}$ flasks and cultured overnight before transfection. The cells were transiently transfected using linear polyethylenimine reagent (Polysciences Inc., USA).

\subsection{BRET $T^{2}$ assay}

A BRET ${ }^{2}$ saturation assay was performed as previously described [7]. Briefly, $48 \mathrm{~h}$ after transfection, HEK293T cells transiently transfected with constant $(1 \mu \mathrm{g})$ or increasing amounts $(0.25-9 \mu \mathrm{g})$ of plasmids encoding for FGFR1 $1^{\text {Rluc }}$ and FGFR $1{ }^{\text {GFP2 }}$, respectively, were rapidly washed twice in PBS, detached, and resuspended in the same buffer. For BRET ${ }^{2}$ measurement, coelenterazine-400a, also known as DeepBlue ${ }^{\mathrm{TM}} \mathrm{C}$ substrate, was added at a final concentration of $5 \mu \mathrm{M}$, and readings with the POLARstar Optima plate reader (BMG Labtechnologies, Offenburg, Germany).

For concentration-response and kinetic BRET ${ }^{2}$ experiments, HEK293T cells were transiently transfected at a constant ratio $(1: 2)$ of FGFR1 ${ }^{\text {Rluc }} /$ FGFR $1^{\text {GFP2 }}$ in presence of heparin $(0.5 \mu \mathrm{M})$. Cells were treated with the indicated FGF ligand concentration or vehicle for 2 min before BRET $^{2}$ measurement. FGF ligand-promoted
$\mathrm{BRET}^{2}$ was calculated by subtracting the $\mathrm{BRET}^{2}$ ratio obtained in the absence of the FGF ligand from that obtained in the presence of the FGF ligand. In the case of kinetic measurements, coelenterazine-400a was added after the FGF ligand ( $30 \mathrm{ng} / \mathrm{ml}$ ), just before BRET $^{2}$ measurements. In each experiment, the specificities of FGFR1/FGFR1 interactions were assessed by comparison with cells expressing FGFR1 ${ }^{\text {GFP2 }}$ alone. As another negative control were used cells individually expressing FGFR $1^{\text {Rluc }}$ that were mixed prior to exposition to coelenterazine-400a with cells individually expressing FGFR1 ${ }^{\text {GFP2 }}$.

\subsection{ERK1/2 phosphorylation assay}

To determine mitogen-activated kinase activity (ERK1/2) in response to FGFs, we used the In-cell Western blot as described [8]. Briefly, HEK293T cells transiently expressing FGFR1 were seeded onto poly-D-lysine-coated 96-well plates (Corning, Corning, NY) grown to $70 \%$ confluence. After $24 \mathrm{~h}$ incubation in serum-free medium, cells were treated or not for 2 min with FGF ligands (30 ng/ $\mathrm{ml}$ ). Cells were washed and fixed with $3.7 \%$ paraformaldehyde for $10 \mathrm{~min}$ at room temperature, washed, blocked for $90 \mathrm{~min}$ in LI-COR Odyssey Blocking Buffer ${ }^{\circledR}$ (LI-COR Biosciences, UK) and then incubated overnight at $4{ }^{\circ} \mathrm{C}$ with primary monoclonal mouse antiphospho-ERK1/2 antibody (Sigma-Aldrich, Stockholm, Sweden) (diluted 1/10000). Then, cells were washed and incubated with an infrared secondary antibody (goat anti-mouse, 1:1500, LI-COR Biosciences, UK) for $1 \mathrm{~h}$ in LI-COR Odyssey Blocking Buffer ${ }^{\circledR}$ at room temperature, washed and plates were scanned by the Odyssey infrared scanner. Total cell number was normalized using DRAQ5/Sapphire 700 staining agents.

\subsection{Bioinformatics prediction of the homodimers interface interaction}

Based on a bioinformatics approach, a set of amino acid triplet homologies have been deduced in receptor homodimers that may be responsible for receptor-receptor interactions $[9,10]$. It has been indicated how such triplets of amino acid residues and their 'teams' may be utilized to construct a kind of code that determines (and/or predicts) which receptors should or should not form homodimers. In this study, the FGFR1-FGFR1 homodimer has been analyzed for the existence of a basic set of common triplets in the two participating receptors that may be responsible for the homodimerization. A comparison with the heavy and light chain of IgG has also been made where also these triplet are located (see Supplementary Material 1).

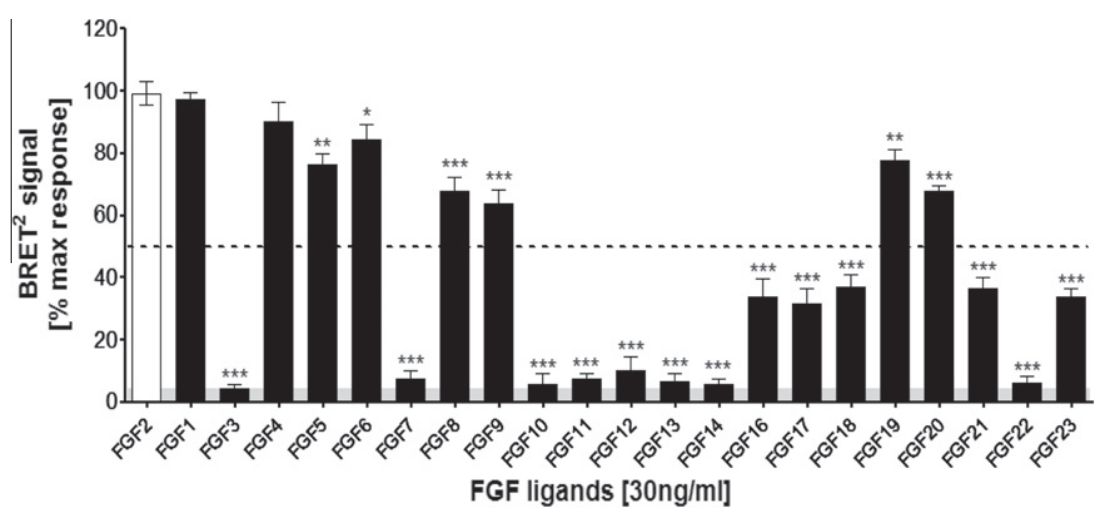

Fig. 1. BRET ${ }^{2}$ studies of FGFR1/FGFR1 homodimer response to FGF ligands in living HEK293T cells. BRET ${ }^{2}$ was measured at a fixed ratio (1:2) of expression levels of FGFR1 $1^{\text {Rluc/ }}$ FGFR $1^{\text {GFP2 }}$ treated with FGF ligands 2 min before BRET ${ }^{2}$ measurement in presence of heparin $(0.5 \mu \mathrm{M})$. Results are expressed as normalized BRET ${ }^{2}$ ratio as described in Section 2. The agonist-induced rise of the BRET ${ }^{2}{ }_{\text {max }}$ value is shown in percent of the vehicle value in absence of FGF agonist ligand. Shaded area represents the $95 \%$ confidence interval of the mean vehicle value. Dashed lines represent $50 \%$ of BRET $^{2}$ max signal. Data represent the mean \pm SEM of four independent experiments performed each in triplicate. *, ${ }^{* *}$ and ${ }^{* * *}$ : Significantly different compared to FGF-2 treatment $(P<0.05, P<0.01$ and $P<0.001$, respectively) by one-way ANOVA followed by Dunnett's comparison post-test. 


\subsection{Statistical analysis}

The number of samples $(n)$ in each experimental condition is indicated in Figure legends. Statistical evaluation of the biochemical data was performed with one-way analysis of variance (ANOVA) followed by Dunnett's comparison post-test or two-way ANOVA followed by Bonferroni comparison post-test. The $P$ value 0.05 and lower was considered significant. Pearson productmoment correlation coefficient was use to determine lineal relation between two quantitative variable $\left(\mathrm{BRET}^{2}{ }_{\max }\right.$ signal and ERK1/2 activity).

\section{Results}

3.1. Differential increases in formation of FGFR1 homodimer upon FGF agonist ligand treatment based on the BRET ${ }^{2}$ technique

The effect of FGF agonist ligand treatment was studied on FGFR1/FGFR1 homodimer formation by means of BRET ${ }^{2}$ analysis. HEK293T cells were transiently co-transfected at a constant ratio $(1: 2)$ of FGFR1 ${ }^{\text {Rluc }} /$ FGFR1 ${ }^{\text {GFP2 }}$. As a positive control were used cells expressing a $\mathrm{GFP}^{2}$-Rluc tandem fusion protein. A mixture of cells expressing singly FGFR ${ }^{\text {Rluc }}$ or FGFR ${ }^{\mathrm{GFP} 2}$ was used as a negative control. Cells were treated with the indicated high FGF agonist ligand concentration $(30 \mathrm{ng} / \mathrm{ml})$, expected to produce a maximal activation of FGFR1 [11], or vehicle for 2 min before BRET ${ }^{2}$ measurements. A positive BRET ${ }^{2}$ signal was obtained from the transfer of energy between FGFR ${ }^{\text {Rluc }}$ and FGFR ${ }^{G F P 2}$. The FGF agonist ligand treatment with FGF-1, $-2,-4,-5,-6,-8,-9,-19$ and -20 led to an increase above $50 \%$ of the basal value in the BRET ${ }^{2}$ signal. With the exception of FGF-1 the other FGF agonist ligand treatments showed significantly reduced increases in the BRET ${ }^{2}$ vs FGF-2 treatment (Fig. 1). The FGF agonist ligand treatment with FGF-16, $-17,-18,-21$ and -23 led to a further reduction of the increase in the BRET $^{2}$ signal as seen from the modest rise of this signal in the range of $30-40 \%$ (Fig. 1). The remaining FGF agonist ligands FGF-3, -7, -10, -11, -12, $-13,-14$ and -22 produced only weak non-significant increases of the BRET $^{2}$ values in the order of $10 \%$ (Fig. 1).

\subsection{Different potencies and kinetics of FGF agonist ligands in producing FGFR1/FGFR1 homodimers based on the BRET ${ }^{2}$ technique}

Concentration-response curves with FGF-2, FGF-4, FGF-8, FGF9, FGF-10, FGF-19 and FGF-20 were performed on the development BRET $^{2}$ signal in HEK293T cells. The cells were transiently cotransfected at a constant ratio (1:2) of FGFR $1^{\text {Rluc }} /$ FGFR $1^{\text {GFP2 }}$ and treated with these FGF agonist ligands as indicated for $2 \mathrm{~min}$ before BRET $^{2}$ measurement. A representative figure of the results from FGF agonist ligand treatments is shown (Fig 2A, details in Supplementary Material 2). As seen in Fig 2A, FGF-2 treatment showed the highest potency to produce a rise of the $\mathrm{BRET}^{2}$ value. The other FGF agonist ligands tested shifted the curves of the rise of $\mathrm{BRET}^{2}$ signal to the right indicating a reduced potency vs FGF-2 to promote FGFR1 homodimer formation compared to FGF-2 (Fig. 2A). FGF-8, FGF-9 and FGF-20 significantly increased the EC50 values (Fig. 2A and Supplementary Material 2). As also shown in Fig 2A, FGF-10 was unable to promote a rise of the BRET ${ }^{2}$ signal at low concentrations. At a high concentration of FGF-10 a weak $\mathrm{BRET}^{2}$ signal developed.

The kinetics of the FGFR1 ${ }^{\text {Rluc }} /$ FGFR1 $1^{\text {GFP2 }}$ interaction after FGF agonist treatment was also studied in transiently transfected HEK293T cells using BRET ${ }^{2}$ assay over a period of $30 \mathrm{~min}$. As shown in Fig. 2B, FGF-2 and FGF-4 treatments showed no significant change of the $\mathrm{BRET}^{2}$ value over the first $10 \mathrm{~min}$. Also FGF-19
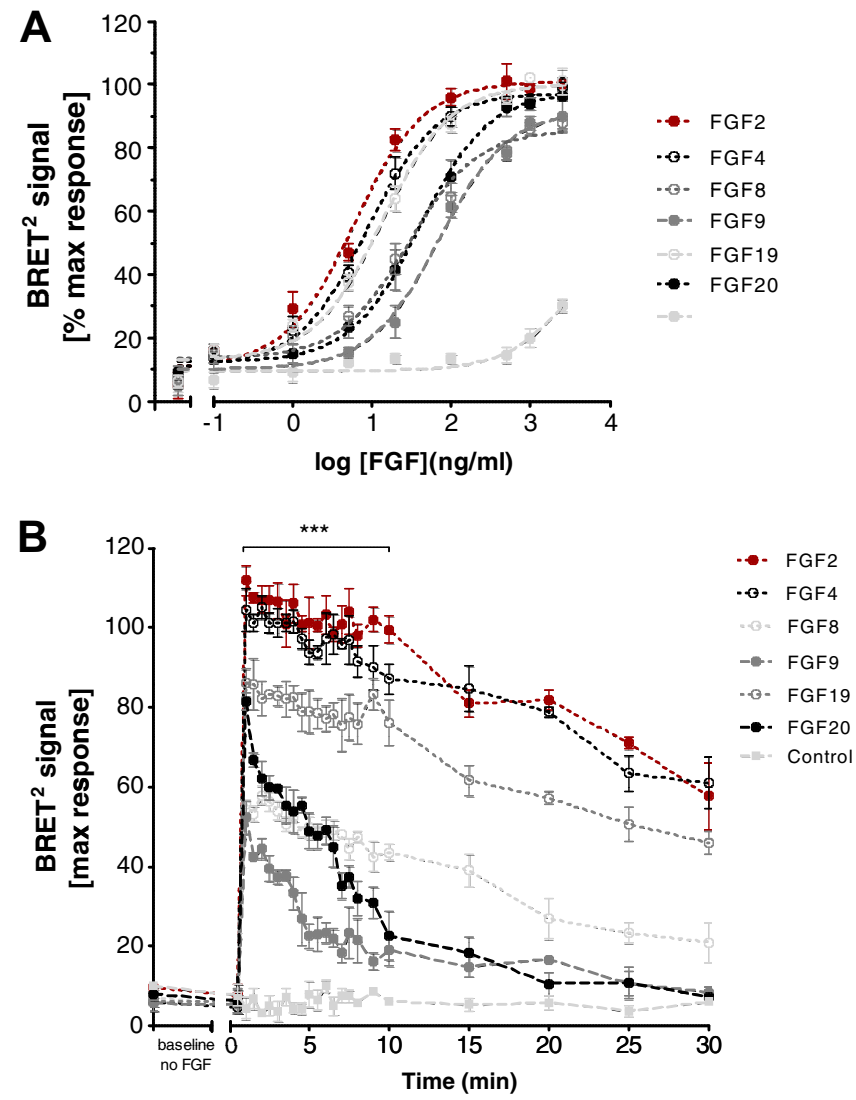

Fig. 2. BRET ${ }^{2}$ studies of FGFR $1^{\text {Rluc }}$ interactions with FGFR1 ${ }^{\text {GFP2 }}$ in living HEK293T cells. BRET ${ }^{2}$ was measured at a fixed ratio $(1: 2)$ of expression levels of FGFR1 ${ }^{\text {Rluc }}$ / FGFR $1^{\text {GFP2 }}$ in presence of heparin $(0.5 \mu \mathrm{M})$. (A) Concentration-response curves of the effects of FGFs on FGFR1/FGFR1 homodimer formation. Cells expressing FGFR1 ${ }^{\text {Rluc }}$ and FGFR $1^{\mathrm{GFP} 2}$ were stimulated in the presence or absence of different concentrations of the FGF ligands shown. Results are expressed as normalized $\mathrm{BRET}^{2}$ ratio with the agonist-induced rise of the $\mathrm{BRET}^{2}{ }_{\max }$ value taken in\% of the value in the absence of FGF ligand as described in Section 2. Data represent the mean \pm SEM of five independent experiments performed each in duplicate. (B) Kinetics of FGFR1 $1^{\text {Rluc }} /$ FGFR1 ${ }^{\text {GFP2 }}$ homodimer formation after the addition of the each FGF agonist ligand at time 0 . Similar experiments were carried out on cells without treatment (vehicle). Mean \pm SEM of five independent experiments performed each in triplicate. The slope value during the first exposure time period $(0-10 \mathrm{~min})$ is $-0.25 \pm 0.44$ for FGF- 2 and $-0.66 \pm 0.19$ and $-1.39 \pm 0.30$ for FGF- 9 and FGF-20 respectively. Linearity was observed in the slopes evaluated with $R^{2}=0.90,0.80$ and 0.70 for FGF-2, -9 and -20 respectively. ${ }^{* * *}$ : The slopes for FGF-9 and FGF-20 are significantly different from the one after FGF-2 treatment in the period of 0-10 min by two-way ANOVA $(P<0001)$ followed by Bonferroni comparison post-test.

although with reduced BRET $^{2}$ max value showed similar results. By contrast, treatment with FGF-9, -20 and to a lesser extent with FGF-8 resulted in a rapid reduction of the BRET ${ }^{2}$ signal with time (Fig. 2B). A marked reduction of the $\mathrm{BRET}^{2}$ signal took place during the first 10 min after FGF-9, and FGF-20 treatment which was significantly different compared with FGF-2 and FGF-4 treatment.

\subsection{Differential increases in ERK1/2 phosphorylation levels after activation of FGFR1 with FGF agonist ligand treatment}

The FGFR1 mediated rise in ERK1/2 phosphorylation levels was studied upon treatment with the same high concentration of FGF agonist ligands as in the BRET ${ }^{2}$ experiments. The findings showed a marked increase in the ERK1/2 phosphorylation levels (maximal response shown in\% of vehicle) after treatment with FGF-1, -2, -4, $5,-6,-19$, which significantly correlated with our BRET ${ }^{2}$ results (Fig. 3 and Supplementary Material 3). A modest rise of ERK1/2 phosphorylation levels was found with FGF-8, -9 and -20 which 


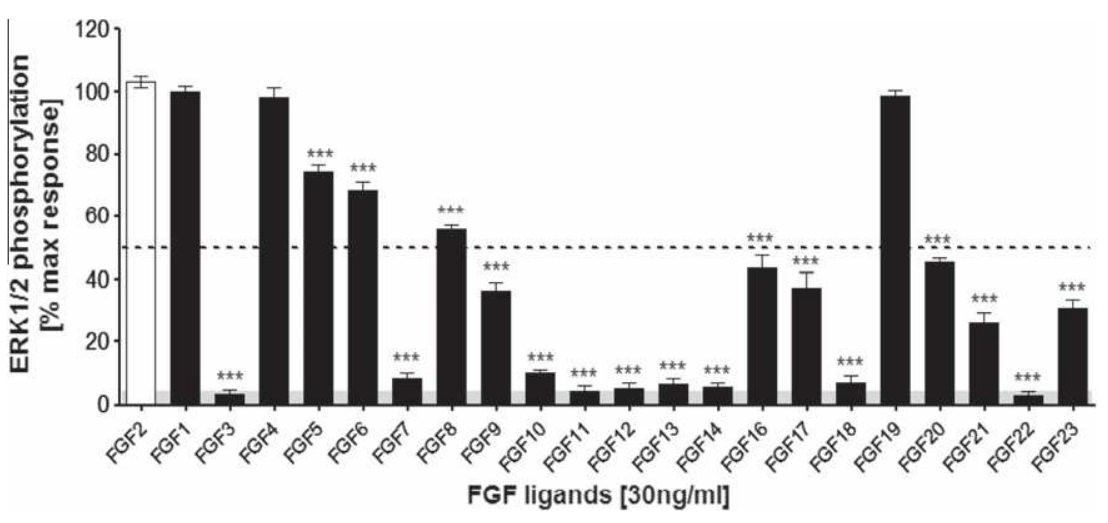

Fig. 3. ERK1/2 of FGFR1 in response to activation by FGF agonist ligands. HEK293T cells transiently expressing FGFR1 were rendered quiescent by serum starvation overnight prior to ERK1/2 phosphorylation experiments. Subsequently, an additional $2 \mathrm{~h}$ pre-incubation step in fresh serum-free medium was performed to minimize basal activity before the cells were challenged with the FGF agonist ligands. Cells were treated with the indicated FGF agonist ligand or vehicle for 2 min. Phosphorylated-ERK1/2 was measured using In-Cell Western blot as described in Section 2. The rise of phospho-ERK1/2 levels is expressed as a percent of the vehicle value. Shaded area represents the 95\% confidence interval of the mean vehicle value. Dashed lines represent $50 \%$ of the maximal phosphorylated-ERK1/2 value. Data represent the mean \pm SEM of three independent experiments performed each in triplicate. ${ }^{* * *}$ : Significantly different compared to FGF-2 treatment $(P<0.001)$ by one-way ANOVA followed by Dunnett's comparison posttest.

also correlated with the modest increase in BRET ${ }^{2}$ levels (Fig. 3 ). The other agonist ligands FGF-3, -7, -10, -11, -12, -13, -14 and 22, did not produce a significant increase in ERK1/2 phosphorylation levels (Fig. 3) as expected from their failure to produce a rise of the BRET $^{2}$ signal (Fig. 1). The findings reveal a convincing and significant correlation between the rise of the BRET ${ }^{2}$ signal in response to FGF agonist ligands and ERK phosphorylation (Supplementary Material 3).

\subsection{Bioinformatic analysis}

The results are summary in Supplementary Material 1, shown that the pro-triplet SSS (Ser-Ser-Ser) is located in both extracellular immunoglobulin domain as well as in the intracellular juxtatransmembrane domain. Furthermore, the pro-triple YGS (TyrGly-Ser) has been demonstrated in the extracellular immunoglobulin domain II. These two pro-triplets were also demonstrated in the heavy and light variable chain of the IgG and also in the heavy variable of the $\operatorname{IgG} \mathrm{M}$, which also participate in protein-protein interaction.

\section{Discussion}

Homodimerization of FGFRs is produced via binding of the FGF agonist ligand/heparin complex to the extracellular binding pocket which leads to trans-phosphorylation processes mediating the different FGFR signaling cascades [12,13]. In this paper we have for the first time studied the extent to which the 23 different types of FGF agonist ligands produce FGFR1 homodimerization determined with BRET $^{2}$ technique and how this property is linked to an increase in the ERK1/2 phosphorylation levels in the HEK293T cells. A highly significant correlation was demonstrated between the FGF agonist ligand induced\% rise of the BRET ${ }^{2}$ max value and the\% rise of the maximal ERK1/2 phosphorylation levels (Fig. 4). Thus, it is likely that the FGFR1 homodimerization is linked at least to the activation of the MAPK pathway.

Indications has been obtained in Bioinformatic analysis (Supplementary Material 1) that pro-triplets SSS (Ser-Ser-Ser) and YGS (Tyr-Gly-Ser) may participate as a guide mechanism to help clasp parts of the homodimer receptor interface at the level of extracellular domain and in the case of SSS also in the intracellular domain position [10]. Further analysis will be established if this hypothesis can be verified.

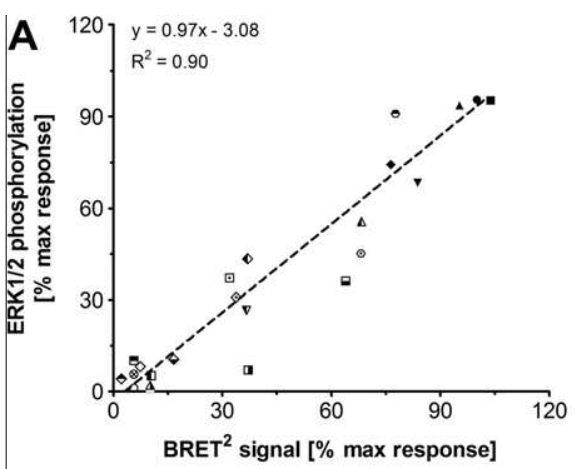

- FGF-2 $\bullet$ FGF-13

- FGF-1 D FGF-12

- FGF-3 FGF-14

- FGF-4 FGF-16

- FGF-5 a FGF-17

- FGF-6 a FGF-18

- FGF-7 $\quad$ FGF-19

$\triangle$ FGF-8 $\odot$ FGF-20

- FGF-9 $v$ FGF-21

- FGF-10 \ FGF-22

- FGF-11 $\odot$ FGF-23

B

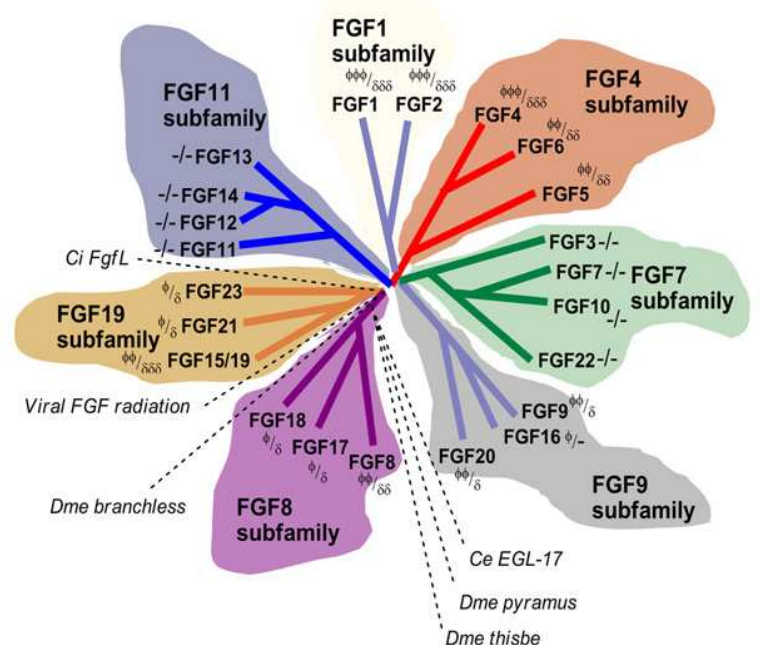

Fig. 4. (A) Correlation between mean BRET ${ }^{2}$ max signal and mean ERK $1 / 2$ phosphorylation levels after activation of FGFR1 with different FGF agonist ligand treatment. Coefficient of determination $R^{2}=0.90$. (B) Schematic representation of the evolutionary tree of the FGF gene network adapted from [11] showing that the FGF agonist ligands in each subfamily have similar characteristics with regard to their ability to increase FGFR1 homodimerization and ERK1/2 phosphorylation. - no effect, $\phi$ and $\delta$ low increase (between $0-25 \%$ ), $\phi \phi$ and $\delta \delta$ moderate increase (between $25-5 \phi 0 \%$ ), $\phi \phi \phi$ and $\delta \delta \delta$ high increase (between $50-100 \%$ ).

There are also marked differences among FGF agonist ligands not only in terms of efficacy to produce FGFR1 homodimerization as seen from the $\mathrm{BRET}^{2}{ }_{\text {max }}$ values but also in their potency to 
produce FGFR1 homodimerization. Among the FGF ligands that produce a significant increased in the BRET ${ }^{2}$ signal and ERK1/2 phosphorylation FGF-1, -2, 4, -5, -6 and -19 . FGF-8, FGF-9 and FGF-20 showed significantly reduced potencies VS FGF-2 in addition to having a reduced efficacy vs FGF-2 as seen from the BRET $^{2}$ max value.

Still another difference among the FGF agonist ligands was in their temporal dynamics to produce and maintain the rise of their BRET $^{2}$ max values. Again, FGF-9 and FGF-20 differed from FGF-2 and also FGF-4 in failing to maintain their BRET $^{2}$ max values over the first 10 min period. Instead, a marked reduction of their maximal values took place. This may reflect instability of the FGFR1 homodimers formed upon activation with these two agonist ligands leading to an increase in their dissociation rate. Taken together the various FGF agonist ligands of the FGF family show substantial differences in producing and maintaining FGFR1 homodimers in living cells, which can help explain their differential biologic effects as illustrated here on the MAPK pathway.

A major result of this study is also that the FGF agonist ligands belonging to the same subfamily of the FGF gene family [14] have similar properties in terms of ability to produce and maintain FGFR1 homodimers and to increase MAPK signaling. Thus, the evolutionary relationship within this gene family appears to have a distinct functional relevance.

Taken together, homodimerization of FGFR1 appear to be a fundamental mechanism for the agonist activity of all FGF ligands at least in the case of the MAPK signaling.

\section{Acknowledgments}

The Torsten and Ragnar Söderberg Foundation, Stockholm, Sweden have supported this work. A.O.T. has not received any support for this work.

\section{Appendix A. Supplementary data}

Supplementary data associated with this article can be found, in the online version, at doi:10.1016/j.bbrc.2011.05.085.

\section{References}

[1] V.P. Eswarakumar, I. Lax, J. Schlessinger, Cellular signaling by fibroblast growth factor receptors, Cytokine Growth F. R. 16 (2005) 139-149.

[2] M. Mohammadi, S.K. Olsen, O.A. Ibrahimi, Structural basis for fibroblast growth factor receptor activation, Cytokine Growth F. R. 16 (2005) 107-137.

[3] A. Yayon, M. Klagsbrun, J.D. Esko, P. Leder, D.M. Ornitz, Cell surface heparinlike molecules are required for binding of basic fibroblast growth factor to its high affinity receptor, Cell 64 (1991) 841-848.

[4] T. Miki, D.P. Bottaro, T.P. Fleming, C.L. Smith, W.H. Burgess, A.M. Chan, S.A. Aaronson, Determination of ligand-binding specificity by alternative splicing: two distinct growth factor receptors encoded by a single gene, P. Natl. Acad. Sci. USA 89 (1992) 246-250.

[5] J. Schlessinger, A. Ullrich, Growth factor signaling by receptor tyrosine kinases, Neuron 9 (1992) 383-391.

[6] S.J. Evans, P.V. Choudary, C.R. Neal, J.Z. Li, M.P. Vawter, H. Tomita, J.F. Lopez, R.C. Thompson, F. Meng, J.D. Stead, D.M. Walsh, R.M. Myers, W.E. Bunney, S.J. Watson, E.G. Jones, H. Akil, Dysregulation of the fibroblast growth factor system in major depression, P. Natl. Acad. Sci. USA 101 (2004) 15506-15511.

[7] D.O. Borroto-Escuela, W. Romero-Fernandez, A.O. Tarakanov, F. Ciruela, L.F. Agnati, K. Fuxe, On the existence of a possible A2A-D2-beta-Arrestin2 complex: A2A agonist modulation of D2 agonist-induced beta-arrestin2 recruitment, J. Mol. Biol. 406 (2011) 687-699.

[8] D.O. Borroto-Escuela, K. Van Craenenbroeck, W. Romero-Fernandez, D. Guidolin, A.S. Woods, A. Rivera, G. Haegeman, L.F. Agnati, A.O. Tarakanov, K. Fuxe, Dopamine D2 and D4 receptor heteromerization and its allosteric receptor-receptor interactions, Biochem. Bioph. Res. Co. 404 (2011) 928-934.

[9] D.O. Borroto-Escuela, W. Romero-Fernandez, A.O. Tarakanov, M. Gomez-Soler F. Corrales, D. Marcellino, M. Narvaez, M. Frankowska, M. Flajolet, N. Heintz, L.F. Agnati, F. Ciruela, K. Fuxe, Characterization of the A2AR-D2R interface: focus on the role of the C-terminal tail and the transmembrane helices, Biochem. Bioph. Res Co. 402 (2010) 801-807.

[10] A.O. Tarakanov, K.G. Fuxe, Triplet puzzle: homologies of receptor heteromers, J. Mol. Neurosci. 41 (2010) 294-303.

[11] M. Garcia-Maya, A.A. Anderson, C.E. Kendal, A.V. Kenny, L.C. Edwards-Ingram, A. Holladay, J.L. Saffell, Ligand concentration is a driver of divergent signaling and pleiotropic cellular responses to FGF. J. Cell. Physiol. 206 (2006) 386-393.

[12] T. Spivak-Kroizman, M.A. Lemmon, I. Dikic, J.E. Ladbury, D. Pinchasi, J. Huang, M. Jaye, G. Crumley, J. Schlessinger, I. Lax, Heparin-induced oligomerization of FGF molecules is responsible for FGF receptor dimerization activation and cell proliferation, Cell 79 (1994) 1015-1024.

[13] M.A. Lemmon, J. Schlessinger, Regulation of signal transduction and signal diversity by receptor oligomerization, Trends Biochem. Sci. 19 (1994) 459463

[14] N. Itoh, D.M. Ornitz, Evolution of the FGF and FGFR gene families, Trends Genet. 20 (2004) 563-569. 